\title{
Sensitivity of Cross Sections for Elastic Nucleus-Nucleus Scattering to Halo Nucleus Density Distributions
}

\author{
G. D. Alkhazov, V. V. Sarantsev \\ Petersburg Nuclear Physics Institute, Gatchina, RUSSIA
}

In order to clear up the sensitivity of the nucleus-nucleus scattering to the nuclear matter distributions of exotic halo nuclei, we have calculated differential cross sections for elastic scattering of the ${ }^{6} \mathrm{He}$ and ${ }^{11} \mathrm{Li}$ nuclei on several nuclear targets at the energy of $0.8 \mathrm{GeV} /$ nucleon with different assumed nuclear density distributions in ${ }^{6} \mathrm{He}$ and ${ }^{11} \mathrm{Li}$.

Comments: 14 pages, 7 figures. Submitted to Proceedings of the 61 International Conference "Nucleus-2011" on the Problems of the Nuclear Spectroscopy and the Atomic Nuclear Structure, Sarov Nijzegorodskaya district, October 10-14, 2011.

Report: Petersburg Nuclear Physics Institute preprint PNPI-2874,10(2011)

Category: Nuclear Theory(nucl-th); High-Energy Physics-Theory (hep-th).

\section{Introduction}

As has been shown in [1,2], the elastic proton-nucleus scattering at intermediate energy in inverse kinematics is an efficient means of studying nuclear matter density distributions in exotic halo nuclei. An analysis of the measured cross sections for small-angular elastic proton-nucleus scattering allows one to determine both the sizes of the nuclear core and the neutron halo. At the same time, as has been shown in [1], the sensitivity of the cross sections for elastic proton-nucleus scattering is not sufficient for obtaining information on the matter distribution of the nuclear far periphery, which contains small amount of the nuclear matter. The authors of paper [3] discuss the sensitivity of the reaction cross sections (that is of the integrated cross sections for all inelastic processes) to the nuclear density distribution at large distances from the nuclear centre. They come to the conclusion that the reaction cross sections for nucleus-nucleus scattering are significantly more sensitive to the nuclear periphery than the reaction cross sections for proton-nucleus scattering. The results of the paper allow us to suppose that the cross sections for elastic nucleus-nucleus scattering are also more sensitive to the nuclear periphery than the cross sections for elastic proton-nucleus scattering. 
If it is really so, then it is reasonable to perform the relevant experiments, for example, at the future nuclear facility FAIR at Darmstadt.

\section{Density distributions}

In the present work, in order to find out the sensitivity of the cross sections for nucleus-nucleus elastic scattering to the nuclear periphery, we have performed calculations of the cross sections for elastic scattering of exotic nuclei ${ }^{6} \mathrm{He}$ and ${ }^{11} \mathrm{Li}$ on protons and nuclear targets ${ }^{4} \mathrm{He},{ }^{9} \mathrm{Be},{ }^{12} \mathrm{C},{ }^{58} \mathrm{Ni},{ }^{90} \mathrm{Zr}$ and ${ }^{208} \mathrm{~Pb}$ at the 800 $\mathrm{MeV} /$ nucleon energy of the incident nuclei, different matter distributions being assumed in the ${ }^{6} \mathrm{He}$ and ${ }^{11} \mathrm{Li}$ nuclei. Theoretical and experimental investigations have shown that the cross sections for proton-nucleus scattering at the intermediate energy ( $\sim 0.5-1.0 \mathrm{GeV} /$ nucleon) can be calculated fairly accurately with the help of the Glauber theory [4]. It should be noted however that calculations of the cross sections for nucleus-nucleus scattering with the exact formula of the Glauber theory is a rather complicated task. In this work, the cross sections for nucleus-nucleus scattering were calculated with the Glauber theory within the "rigid-target" approximation [5], that is at first the amplitude of scattering of one nucleon on the nuclear target was calculated, and then this amplitude was used in the calculations of the scattering of an exotic nucleus consisting of several nucleons. As was shown in [6], the reaction cross sections for scattering of exotic nuclei on nuclear targets calculated within the "rigid-target" approximation are very close to those calculated with the exact Glauber formula.

In the present calculations it was assumed that the ${ }^{6} \mathrm{He}$ and ${ }^{11} \mathrm{Li}$ nuclei consist of the nuclear core of 4 and 9 nucleons, correspondingly in ${ }^{6} \mathrm{He}$ and ${ }^{11} \mathrm{Li}$, and 2 halo neutrons. The matter density distributions in the core were described by Gaussian distributions, whereas the density distributions in the halo were described by a Gaussian for ${ }^{6} \mathrm{He}$ and by a 1p-shell harmonic oscillator-type function for ${ }^{11} \mathrm{Li}$. Spin-independent isospin-avera- ged amplitude of the free nucleon-nucleon $(\mathrm{NN})$ scattering was used, the traditional high-energy parametrization for this amplitude being taken (see [1]) with the following parameters: the total cross section $\sigma_{\mathrm{NN}}=42.5 \mathrm{mb}$, the ratio of the real to imaginary part $\epsilon_{\mathrm{NN}}=-0.18$, and the amplitude slope $\beta_{\mathrm{NN}}=0.2 \mathrm{fm}^{2}$.

Figures 1 and 2 show the ${ }^{6} \mathrm{He}$ matter density distributions applied in the calculations. The solid curve in Fig. 1 corresponds to the nuclear density distribution with a halo: $R_{c}=1.95 \mathrm{fm}, R_{h}=2.88 \mathrm{fm}$ and $R_{m}=2.30 \mathrm{fm}$, where $R_{c}, R_{h}$ and $R_{m}$ are the rms radii of the core, halo and total matter density. The dashed and dotted curves in Fig. 1 correspond to the density distributions without a halo $\left(\mathrm{R}_{\mathrm{c}}\right.$ $=R_{h}$ ), the dashed curve stands for $R_{m}=1.95 \mathrm{fm}$, and the dotted curve stands for $\mathrm{R}_{\mathrm{m}}=2.30 \mathrm{fm}$.

The solid curve in Fig. 2 corresponds to the solid curve in Fig. 1. The halo density distribution in this version of the calculations, as it has been already 


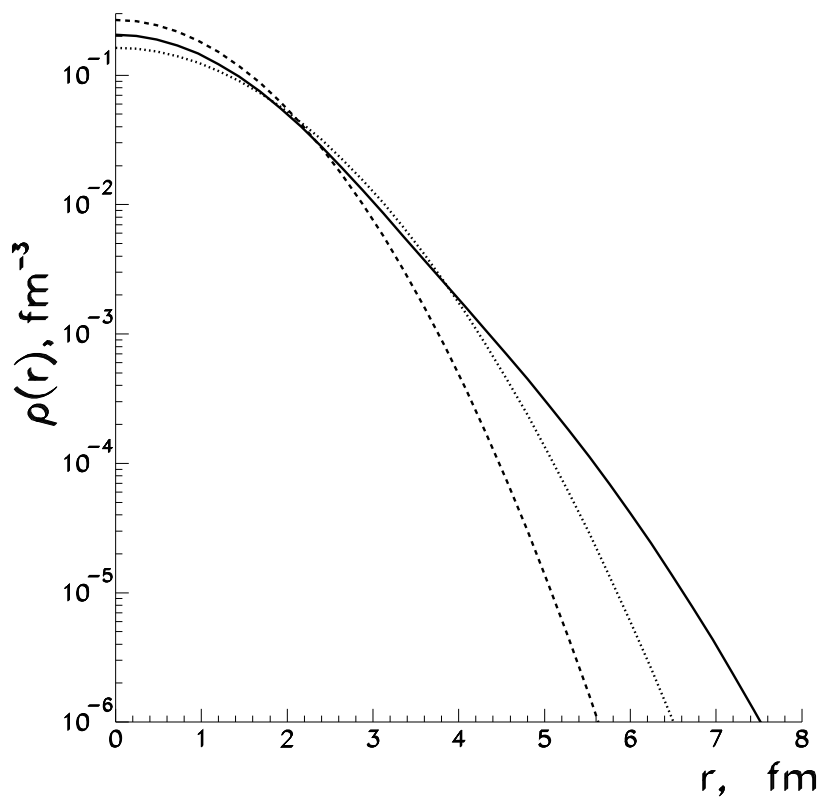

Figure 1: The nuclear matter density distributions in ${ }^{6} \mathrm{He}$ (with a halo structure - solid curve, and without a halo - dashed and dotted curves) applied in the cross sections calculations (for the parameters of the density distributions see the text)

said, is described with a Gaussian distribution. The density in this distribution decreases with increasing the distance $r$ from the nuclear centre faster than it is predicted by theory. The dashed curve in Fig. 2 shows the ${ }^{6} \mathrm{He}$ density distribution where we have added a "tail", which decreases exponentially with the radius $r$ increasing. The resulting density distribution with this tail corresponds to the theoretical nuclear matter distribution FC of Ref. [7]. The halo rms radius in this case is equal to $R_{h}^{\prime}=3.34 \mathrm{fm}$. Figure 2 shows also by the dotted curve the ${ }^{6} \mathrm{He}$ density distribution without a tail, but with the increased halo radius: $\mathrm{R}_{\mathrm{h}}=$ $\mathrm{R}_{\mathrm{h}}^{\prime}=3.34 \mathrm{fm}$.

Figure 3 shows the ${ }^{11} \mathrm{Li}$ density distributions used in the calculations. The solid curve in Fig. 3 corresponds to the density distribution with a halo (however without a tail): $R_{c}=2.50 \mathrm{fm}, R_{h}=5.86 \mathrm{fm}, R_{m}=3.37 \mathrm{fm}$. The dotted curve shows the density distribution without a halo $\left(R_{c}=R_{h}, R_{m}=3.37 \mathrm{fm}\right)$, and the dashed curve shows the density distribution with a halo and the tail corresponding to the theoretical density distribution P2 of Ref. [8].

The matter density distribution of the ${ }^{4} \mathrm{He}$ target nucleus was described by a Gaussian distribution with the rms matter radius $R_{m}=1.49 \mathrm{fm}$ [1]. For heavier nuclear targets, the Fermi distribution

$$
\rho(r) \sim\left[1+\mathrm{w}\left(r / \mathrm{R}_{0}\right)\right] /\left\{1+\exp \left[\left(r-\mathrm{R}_{0}\right) / \mathrm{a}\right]\right\},
$$

was used with the following parameters:

$\mathrm{R}_{0}=1.95 \mathrm{fm}, \mathrm{a}=0.60 \mathrm{fm}, \mathrm{w}=0\left(\mathrm{R}_{\mathrm{m}}=2.68 \mathrm{fm}\right)$ for ${ }^{9} \mathrm{Be}[9]$; 


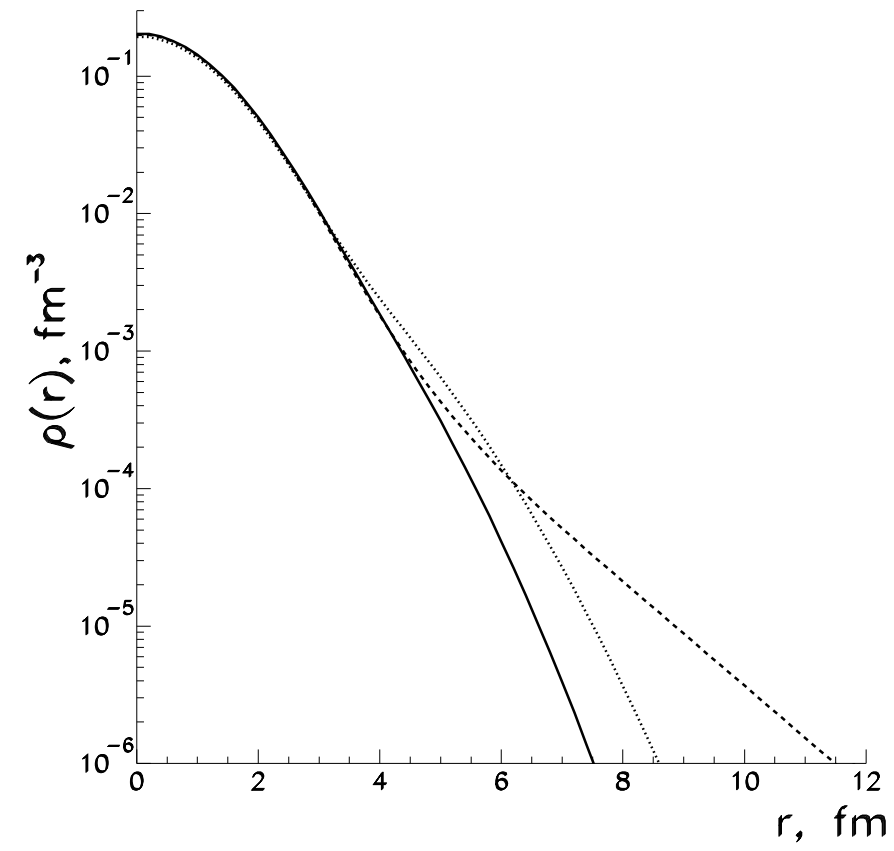

Figure 2: The nuclear matter density distributions in ${ }^{6} \mathrm{He}$ applied in the cross sections calculations, with a density tail - dashed curve, and without a density tail - solid and dotted curves

$\mathrm{R}_{0}=2.12 \mathrm{fm}, \mathrm{a}=0.52 \mathrm{fm}, \mathrm{w}=0\left(\mathrm{R}_{\mathrm{m}}=2.52 \mathrm{fm}\right)$ for ${ }^{12} \mathrm{C}[9]$;

$\mathrm{R}_{0}=3.17 \mathrm{fm}, \mathrm{a}=0.59 \mathrm{fm}, \mathrm{w}=-0.12\left(\mathrm{R}_{\mathrm{m}}=3.15 \mathrm{fm}\right)$ for ${ }^{28} \mathrm{Si}[10]$;

$\mathrm{R}_{0}=4.23 \mathrm{fm}, \mathrm{a}=0.55 \mathrm{fm}, \mathrm{w}=-0.13\left(\mathrm{R}_{\mathrm{m}}=3.76 \mathrm{fm}\right)$ for ${ }^{58} \mathrm{Ni}[11]$;

$\mathrm{R}_{0}=4.99 \mathrm{fm}, \mathrm{a}=0.57 \mathrm{fm}, \mathrm{w}=-0.09\left(\mathrm{R}_{\mathrm{m}}=4.35 \mathrm{fm}\right)$ for ${ }^{90} \mathrm{Zr}[12]$;

$\mathrm{R}_{0}=6.70 \mathrm{fm}, \mathrm{a}=0.54 \mathrm{fm}, \mathrm{w}=-0.06\left(\mathrm{R}_{\mathrm{m}}=5.54 \mathrm{fm}\right)$ for ${ }^{208} \mathrm{~Pb}[12]$.

(Note that the used Fermi distributions include the final size of the nucleon). When performing the calculations of the cross sections only the centre-of-mass correlations were taken into account in the many-body density distributions (see [1]). The results of the calculations are presented in Figs. 4-7.

\section{Results and discussion}

Figure 4 shows the calculated cross sections for elastic ${ }^{6} \mathrm{He}-p,{ }^{6} \mathrm{He}^{-}{ }^{4} \mathrm{He},{ }^{6} \mathrm{He}^{12} \mathrm{C}$ and ${ }^{6} \mathrm{He}-{ }^{58} \mathrm{Ni}$ scattering. The solid curves correspond to the calculations with the halo density distributions in ${ }^{6} \mathrm{He}: R_{\mathrm{c}}=1.95 \mathrm{fm}, \mathrm{R}_{\mathrm{h}}=2.88 \mathrm{fm}, \mathrm{R}_{\mathrm{m}}=2.30 \mathrm{fm}$ (see Fig. 1). The dashed and dotted curves correspond to density distributions without a halo $\left(R_{c}=R_{h}\right)$, the dashed curve stands for $R_{m}=1.95 \mathrm{fm}$, and the dotted curve stands for $R_{m}=2.30 \mathrm{fm}$.

It is seen that at small momentum transfers the behaviour of the cross sections is governed by the value of $R_{m}$ - the rms radius of the total nuclear matter distribution. Indeed, the cross sections calculated for the matter density distri- 


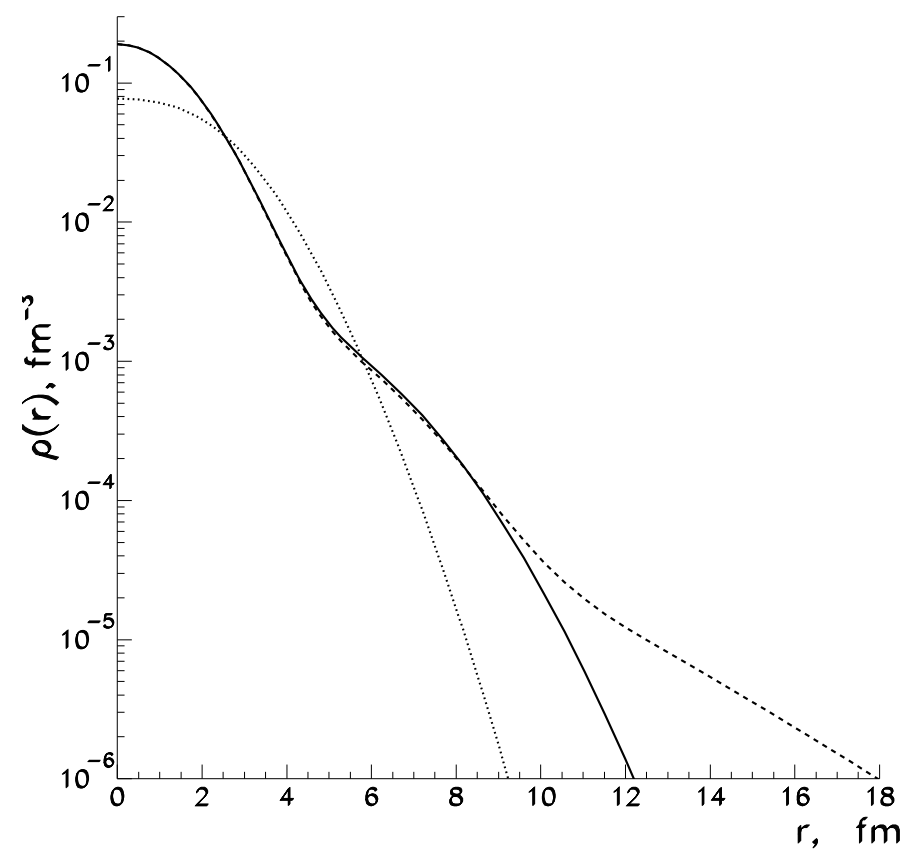

Figure 3: The nuclear matter density distributions in ${ }^{11} \mathrm{Li}$ applied in the cross sections calculations (for the parameters of the density distributions see the text)

butions of different shape but with the same rms matter radius at $-t<0.03$ $(\mathrm{GeV} / \mathrm{c})^{2}$ are practically the same (compare the solid and dotted curves in Fig. 4). At larger values of $|t|$ the cross sections corresponding to these two density distributions become significantly different. A comparison of the calculated cross sections for scattering of the ${ }^{6} \mathrm{He}$ nuclei on different nuclear targets shows that the sensitivity of the nucleus-nucleus cross sections to the shape of the nuclear matter distribution in ${ }^{6} \mathrm{He}$ is not higher than that of the cross section for ${ }^{6} \mathrm{He}-p$ scattering.

To clear up the sensitivity of the cross sections to the nuclear far periphery, we have calculated the relevant cross sections using the nuclear matter densities in ${ }^{6} \mathrm{He}$ with the density tail and without it (Fig. 2). Since the tail contains a very small amount of the total nuclear matter (on the level of 1\%), the effect in the cross sections from the scattering on the nucleons of the tail is so small that it is practically not seen in the cross sections depicted in the logarithmic scale. Therefore, to see in the cross sections the effect of taking into account this tail, we show in Fig. 5 the ratio of the cross sections calculated for the matter density distributions with and without the tail. We see that in the cross section for ${ }^{6} \mathrm{He}-$ nucleus scattering the size of the effect of taking into account the ${ }^{6} \mathrm{He}$ density tail is approximately the same as that in the cross section for ${ }^{6} \mathrm{He}-p$ scattering. Figure 5 also shows (by the dotted curves) the ratio of the cross sections calculated for the ${ }^{6} \mathrm{He}$ density without the tail with the rms halo radii $\mathrm{R}_{\mathrm{h}}=2.88 \mathrm{fm}$ and $\mathrm{R}_{\mathrm{h}}=$ $3.34 \mathrm{fm}$ (this halo radius corresponds to the density version with the tail). 


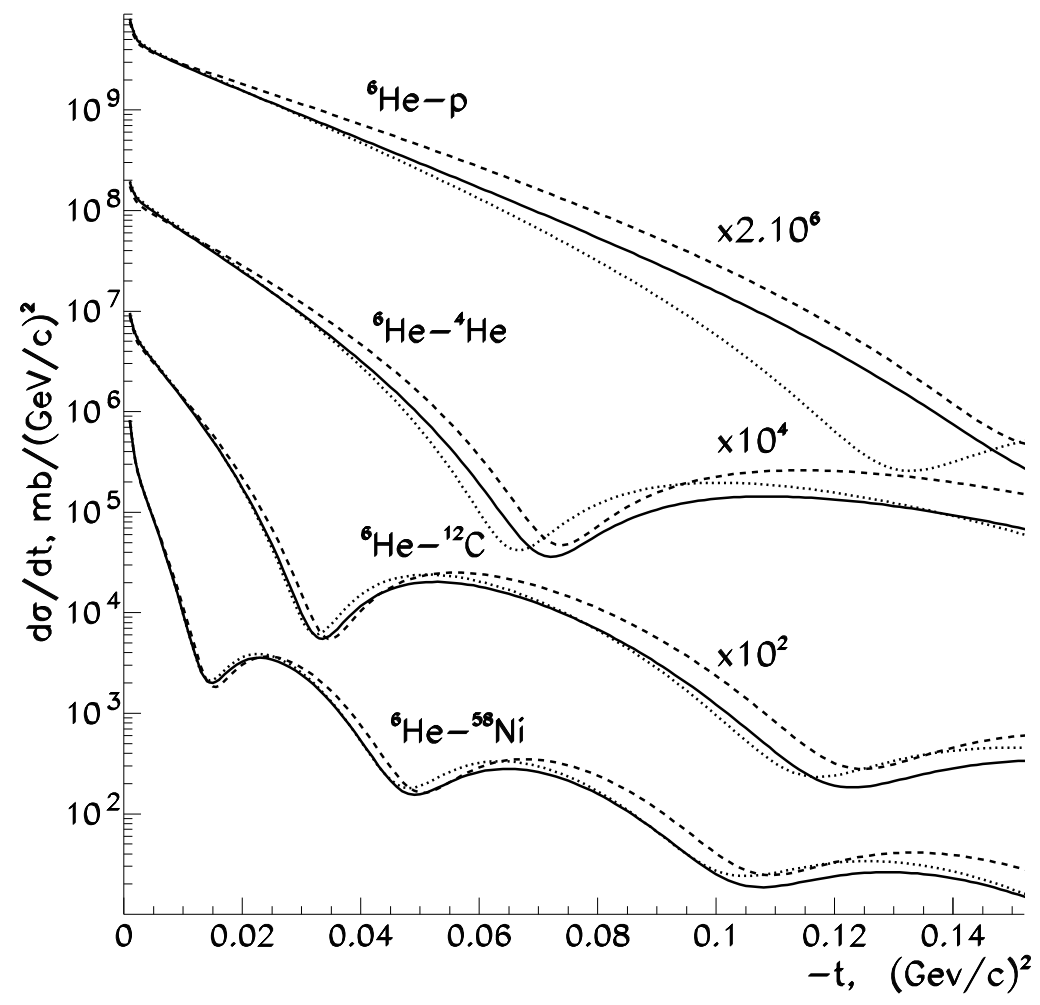

Figure 4: The calculated cross sections for scattering of ${ }^{6} \mathrm{He}$ on protons and nuclear targets ${ }^{4} \mathrm{He},{ }^{12} \mathrm{C}$ and ${ }^{58} \mathrm{Ni}$ at the energy of $0.8 \mathrm{GeV} /$ nucleon as functions of the fourmomentum transfer squared $-t$

We see that in the cases of ${ }^{6} \mathrm{He}-p$ and ${ }^{6} \mathrm{He}-{ }^{12} \mathrm{C}$ scattering the effects in the cross sections due to taking into account the density "tail" and due to the corresponding increase of the halo radius in the density without the "tail" are significantly different. However, in the case of ${ }^{6} \mathrm{He}$ scattering on heavy nuclei (here ${ }^{208} \mathrm{~Pb}$ ) the difference between the cross sections calculated with these two density versions becomes smaller (for ${ }^{6} \mathrm{He}^{208} \mathrm{~Pb}$ scattering the solid curve in Fig. 5 has the form similar to that of the dotted curve). Therefore it would be difficult in this case to determine whether the density distribution does have a "tail" or does not.

Figure 6 shows the calculated cross sections for elastic ${ }^{11} \mathrm{Li}-p,{ }^{11} \mathrm{Li}^{-12} \mathrm{C}$ and ${ }^{11} \mathrm{Li}-{ }^{90} \mathrm{Zr}$ scattering. The solid curves correspond to the calculations for the ${ }^{11} \mathrm{Li}$ nuclear matter density distribution with a halo (but without a "tail"): $\mathrm{R}_{\mathrm{c}}=$ $2.50 \mathrm{fm}, \mathrm{R}_{\mathrm{h}}=5.86 \mathrm{fm}, \mathrm{R}_{\mathrm{m}}=3.37 \mathrm{fm}$ (see Fig. 3 ). The dashed curves correspond to the calculations for the density distribution without a halo but with the same rms matter radius: $R_{c}=R_{h}=R_{m}=3.37 \mathrm{fm}$. We see that the sensitivity of the cross sections to the shape of the ${ }^{11} \mathrm{Li}$ nuclear matter distribution in the case when the target nucleus is heavy (here it is ${ }^{90} \mathrm{Zr}$ ) is smaller than that in the case when the target is light (hydrogen or ${ }^{12} \mathrm{C}$ ). Evidently, it is partly due to a significant contribution to the cross section from the Coulomb scattering in the case of heavy 


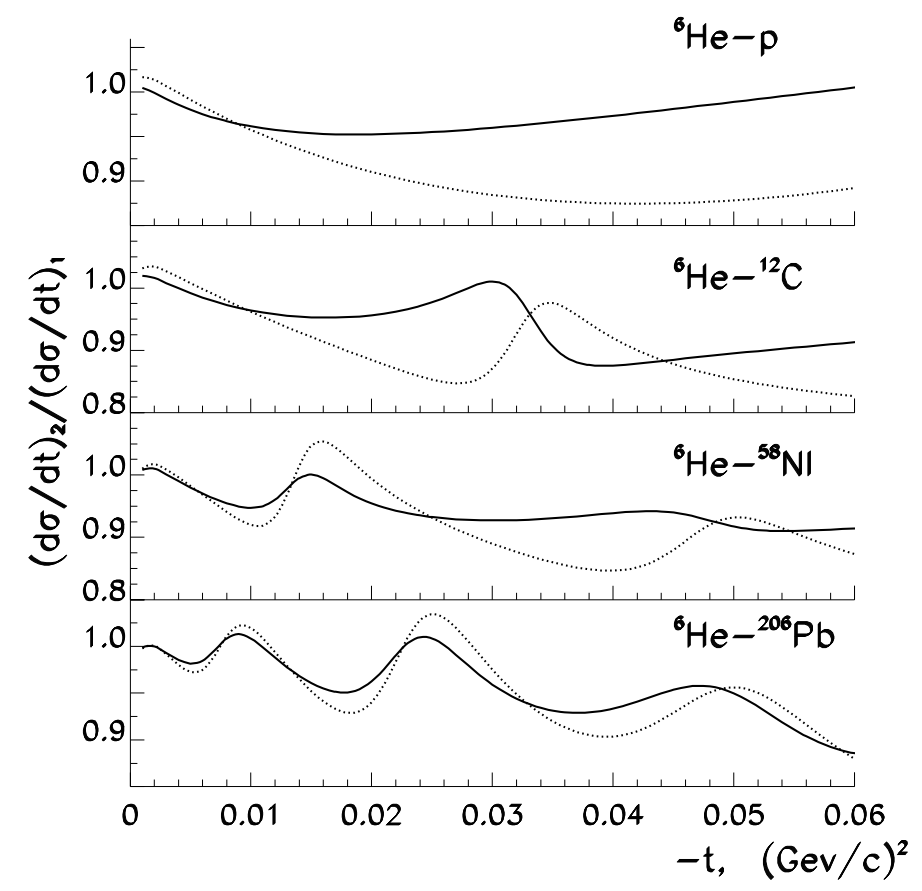

Figure 5: The ratio of the cross sections $(\mathrm{d} \sigma / \mathrm{d} t)_{2}$ for scattering of ${ }^{6} \mathrm{He}$ on protons and nuclear targets ${ }^{12} \mathrm{C},{ }^{58} \mathrm{Ni}$ and ${ }^{208} \mathrm{~Pb}$, calculated with the ${ }^{6} \mathrm{He}$ matter density distribution with a "tail" (see Fig. 2) to the corresponding cross sections $(\mathrm{d} \sigma / \mathrm{d} t)_{1}$, calculated with the distribution without this "tail" (solid curves). Dotted curves stand for the ratios of the cross sections $(\mathrm{d} \sigma / \mathrm{d} t)_{2} /(\mathrm{d} \sigma / \mathrm{d} t)_{1}$, calculated using the ${ }^{6} \mathrm{He}$ matter density distributions without a tail correspondingly for the halo radii $R_{h}=3.34 \mathrm{fm}$ and $R_{h}=$ $2.88 \mathrm{fm}$

nuclear targets (targets with large Z). The dotted curves in Fig. 6 show also the cross sections calculated for the ${ }^{11} \mathrm{Li}$ density distribution with a halo, but without taking into account the contribution from the Coulomb scattering. We see that in the ${ }^{11} \mathrm{Li}-p$ scattering cross section the contribution from the Coulomb scattering is small (this contribution is seen only at very small values of $|t|<0.005(\mathrm{GeV} / \mathrm{c})^{2}$ and in the region of the diffraction minimum). In the ${ }^{11} \mathrm{Li}^{12} \mathrm{C}$ cross section, the contribution from the Coulomb scattering is more significant, while in the ${ }^{11} \mathrm{Li}$ ${ }^{90} \mathrm{Zr}$ cross section the contribution from the Coulomb scattering is comparable to that of the strong interaction scattering, or even surpasses it.

In order to clear up the sensitivity of the scattering cross sections to the ${ }^{11} \mathrm{Li}$ nuclear far periphery, we have calculated the cross sections for ${ }^{11} \mathrm{Li}$ scattering on several nuclear targets for the ${ }^{11} \mathrm{Li}$ matter density distributions with and without the "tail". Figure 7 presents the results of the calculations. We see that at small momentum transfers $|t|$ the effect in the cross sections from taking into account the "tail" of the ${ }^{11} \mathrm{Li}$ matter distribution for the ${ }^{11} \mathrm{Li}$-nucleus scattering is approximately the same as that for ${ }^{11} \mathrm{Li}-p$ scattering. At larger values of $|t|$, in the regions of diffraction minima, the sensitivity of the cross sections to the nuclear far periphery for ${ }^{11} \mathrm{Li}$-nucleus scattering is somewhat higher than that for 


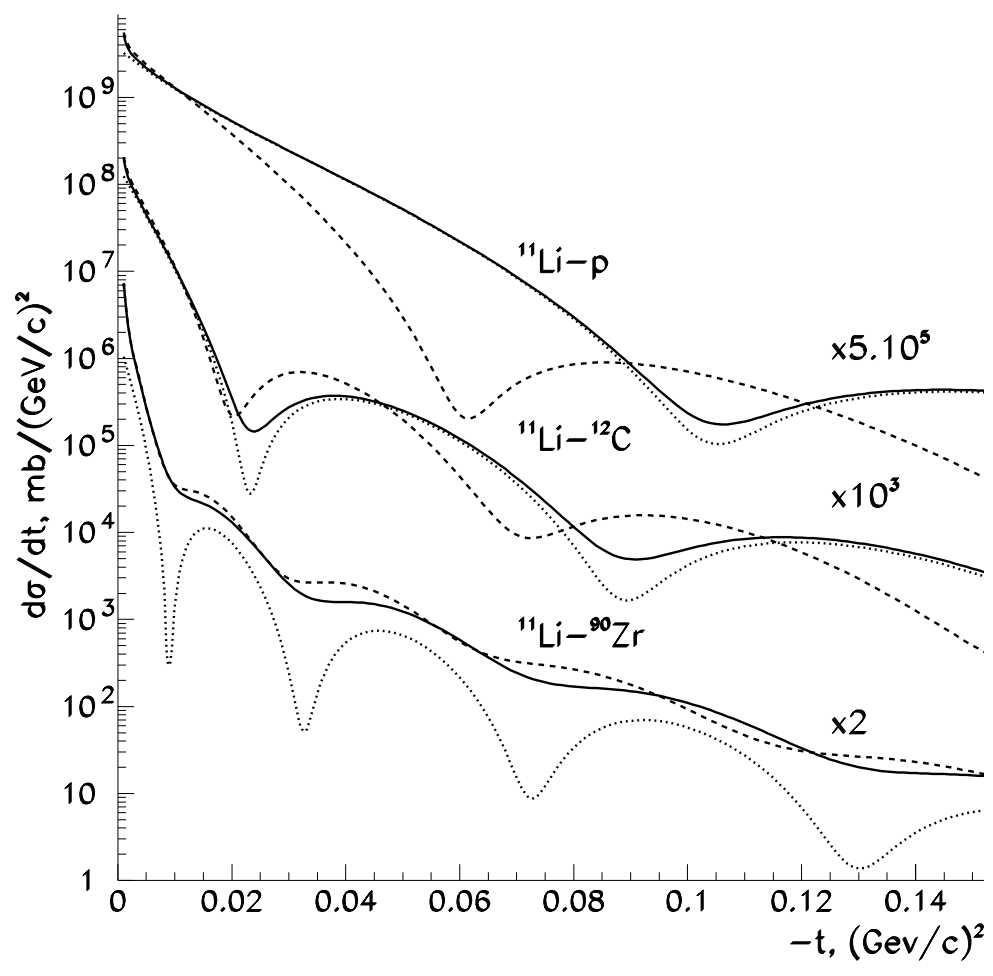

Figure 6: The calculated cross sections for scattering of ${ }^{11} \mathrm{Li}$ on protons and nuclear targets ${ }^{12} \mathrm{C}$ and ${ }^{90} \mathrm{Zr}$

${ }^{11}$ Li-proton scattering.

However, we should bear in mind that the calculated cross sections in the diffraction minima are subject to noticeable uncertainties (of the Coulomb contribution, of the Coulomb-nuclear interference, due to some uncertainties in the ratio of the real-to-imaginary parts of the NN-scattering amplitude and due to some other reasons). Therefore, in the analyses of the measured scattering cross sections with the purpose to study nuclear matter distributions it is desirable not to use the cross sections in the regions of the diffraction minima.

\section{Conclusion}

The whole set of the carried out considerations gives evidence that the sensitivity of the cross sections for nucleus-nucleus scattering to the shape of the nuclear matter distribution of the studied exotic nuclei is of the same order of magnitude as that of the cross sections for nucleus-proton scattering. We do not see marked advantages in using cross sections for elastic nucleus-nucleus scattering with the aim to study nuclear matter distributions in exotic nuclei as compared to using cross sections for nucleus-proton scattering. It should be noted also that both the measurements of the differential cross sections and calculations of the cross sec- 


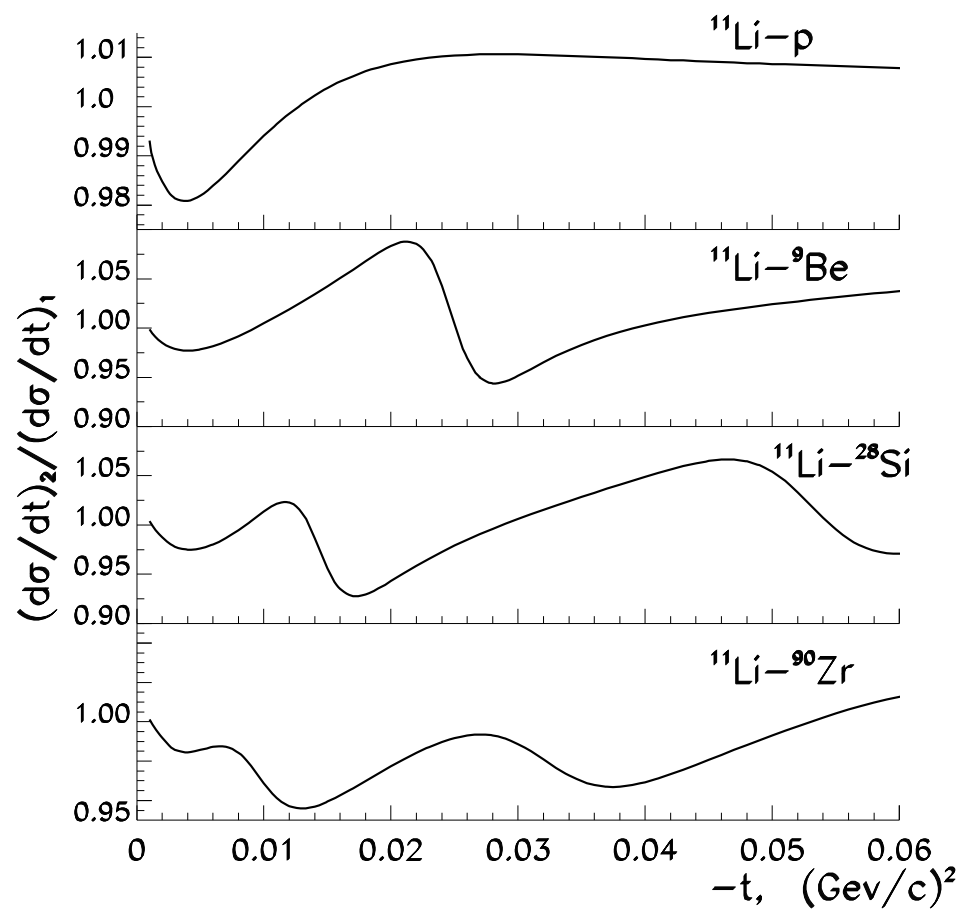

Figure 7: The ratio of the cross sections $(\mathrm{d} \sigma / \mathrm{d} t)_{2}$ for scattering of the ${ }^{11} \mathrm{Li}$ nuclei on hydrogen and nuclear targets ${ }^{9} \mathrm{Be},{ }^{28} \mathrm{Si}$ and ${ }^{90} \mathrm{Zr}$, calculated with the ${ }^{11} \mathrm{Li}$ matter density with a "tail" (Fig. 3), to the corresponding cross sections $(\mathrm{d} \sigma / \mathrm{d} t)_{1}$, calculated without the "tail"

tions with the exact Glauber formula for nucleus-nucleus scattering are noticeably more complicated tasks than the corresponding measurements and calculations for nucleus-proton scattering. We come to the conclusion that performing experiments to measure differential cross sections for elastic nucleus-nucleus scattering with the purpose to study nuclear matter distributions in exotic nuclei is hardly justified.

\section{References}

1. Alkhazov G. D., Dobrovolsky A. V., Egelhof P. et al., Nucl. Phys. A 712, 269(2002).

2. Dobrovolsky A. V., Alkhazov G. D., Andronenko M. N. et al., Nucl. Phys. A 766, 1(2006).

3. Bush M. P., Al-Khalili J. S., Tostovin J. A., Johnson R. C., Phys. Rev. C 53, 3009(1966).

4. Glauber R. J., Lectures in theoretical physics, eds. W. E. Britten and

L. G. Dunham, New York: Interscience, 1959, V. 1. P. 315.

5. Alkhazov G. D., Bauer T., Bertini R. et al., Nucl. Phys. A 280, 365(1977). 
6. Alkhazov G. D. and Lobodenko A. A., Yad. Fiz. V. 70. 1(2007).

7. Al-Khalili J. S., Tostevin J. A., Phys. Rev. C 56, 1846(1998).

8. Thompson I. J., Zhukov M. V., Phys. Rev. C 49, 1904(1994).

9. Alkhazov G. D., Belostotsky S. L., Vorobyov A. A. et al., Yad. Fiz. V. 42. 8(1985).

10. Alkhazov G. D., Belostotsky S. L., Vorobyov A. A. et al., Yad. Fiz. V. 22. 902(1975).

11. Lombard R. M., Alkhazov G. D., Domchenkov O. A., Nucl. Phys. A 360, 233(1981).

12. Alkhazov G. D., Belostotsky S. L., Vorobyov A. A. et al., Yad. Fiz. V. 26. 673(1977). 